\title{
ZEITSCHRIFT \\ FÜR
}

ANGEWANDTE

GEOLOGIE

HERAUSGEGEBEN VON

DER STAATLICHEN GEOLOGISCHEN KOMMISSION

UND DER ZENTRALEN VORRATSKOMMISSION

DER DEUTSCHEN DEMOKRATISCHEN REPUBLIK
AUS DEM INHALT

Zwölf Jahre

Deutsche Demokratische Republik

F. Stammberger

Vorschlïge zur Verbesserung

der hestehenden DDR-Klassifikation

D. Reinhold

Bemerkungen zu den neuen Richtlinien der ZVK über Form und Inhalt

von Vorratsberechnungen

A. A. Amiraslanow, M. B. Borodajewskaja \& M.S. Bessmertnaja

Gesetzmäßigkeiten in der Verteilung endogener Lagerstätten

M. Kraft

Zur erzmikroskopischen Cutersuchung von Auf bereitungsprodukten am Beispiel von Blei-Zink-Errzen des Brander Reviers

D. Andreas \& G. Hecht

Die Induzierte Polarisation als Bohrlochmeßverfahren bei der Buntmetallerkundung

E. Wohlmann

Schnellverfahren zur Analyse einfacher Silikate 